\title{
Qualidade da água utilizada para consumo humano proveniente do Aquífero Alter do Chão em Santarém (Oeste do Pará) e sua relação com a saúde pública
}

Valeria de Sousa Bentes Geraldo Walter de Almeida Neto Marina Smidt Celere Meschede*

Instituto de Saúde Coletiva

Universidade Federal do Oeste do Pará Rua Vera Paz, s/n

Unidade Tapajós)

Bloco Modular Tapajós (BMT), Sala 222

Bairro Salé

Santarém PA Brasil

CEP 68040-255

*Autora correspondente:

marina.meschede@ufopa.edu.br

\section{RESUMO}

O abastecimento de água em Santarém é favorecido pela a ocorrência do aquífero Alter do Chão, cuja captação da água é realizada por poços e distribuída sem tratamento para a população. A expansão urbana acelerada e a falta de serviços de saneamento básico, vem comprometendo a qualidade da água, o que poderá acarretar implicações para a saúde. O objetivo desse trabalho foi avaliar a qualidade microbiológica e físico-química da água de consumo em Santarém e as implicações para a saúde. Selecionou-se 30 poços domiciliares e coletou-se 60 amostras de água durante a estação seca e chuvosa. Os valores foram comparados com as recomendações da Portaria 2914/11 do Ministério da Saúde e verificado diferenças estatísticas nos parâmetros obtidos entre as estações. A maior parte dos parâmetros avaliados estiveram em concordância com a legislação, entretanto, valores de $\mathrm{pH}$ apontaram água ácida o que poderá acarretar perturbações gástricas e intestinais nos consumidores. Em 33\% e $40 \%$ das amostras durante a estação seca e chuvosa, respectivamente, apresentaram nitrato elevado, demostrando deficiências sanitárias. Em 37\% das amostras coletadas durante a estação seca e 33\% durante a chuvosa apresentaram concentrações superiores ao recomendado de alumínio, sendo que estudos tem demonstrado uma relação ainda em debate entre exposição crônica ao alumínio e doenças neurológicas. Não houve diferenças significantes entre os parâmetros nas estações analisadas. Conclui-se que a água consumida nos domicílios, apresenta-se com alguns parâmetros de qualidade em desacordo, evidenciando que a população está exposta a riscos microbiológicos e químicos através do seu consumo.

Palavras-chave: Água Subterrânea. Ecossistema Amazônico. Saúde Humana.

\section{ABSTRACT}

The water supply in Santarém is favoured by the occurrence of the Alter do Chão aquifer, whose water is collected by wells and distributed without treatment to the population. Accelerated urban expansion and the lack of basic sanitation services have been compromising water quality, which may have implications for health. The objective of this work was to evaluate the microbiological and physical-chemical quality of drinking water in Santarém and the implications for health. Thirty household wells were selected and 60 water samples were collected during the dry and rainy season. The values were compared with the recommendations of Ordinance 2914/11 of the Ministry of Health and verified statistical differences in the parameters obtained between the seasons. Most of the parameters evaluated were in agreement with the legislation, however, $\mathrm{pH}$ values pointed to acidic water, which may cause gastric and intestinal disturbances in consumers. In $33 \%$ and $40 \%$ of the samples during the dry and rainy season, respectively, they showed high nitrate, showing sanitary deficiencies. In $37 \%$ of the samples collected during the dry season and $33 \%$ during the rainy season, they presented concentrations higher than the recommended amount of aluminium, and studies have shown a relationship still under debate between chronic exposure to alumini- 
um and neurological disease. There were no statistical differences between the parameters at the stations analysed. It is concluded that the water consumed in the homes, presents with some quality parameters in disagreement, showing that the population is exposed to microbiological and chemical risks through its consumption.

Keywords: Groundwater. Amazonian Ecosystem. Human's Health.

\section{INTRODUÇÃO}

A cidade de Santarém, vem apresentando nas últimas décadas um significativo crescimento demográfico. Segundo dados do Instituto Brasileiro de Geografia e Pesquisa (IBGE), analisados por Cortês (2012), Santarém apresentava uma taxa de urbanização de $45,4 \%$ em 1970 e de $73,3 \%$ em 2010 . Esse processo acelerado de urbanização, reconhecível em toda a região amazônica, não foi acompanhado de uma evolução adequada de infraestrutura e serviços demandados pela população. Santarém é uma cidade que já apresenta alguns dos efeitos negativos da rápida urbanização, caracterizada pelo processo de periferização intensificado nas últimas duas décadas (OLIVEIRA 2008), implicando na existência de problemas de saneamento que podem afetar negativamente a qualidade da água de consumo humano e a saúde da população.

$\mathrm{Na}$ região do Oeste do estado do Pará, o suprimento de água para consumo humano é muito favorecido pela ocorrência do volumoso sistema aquífero Alter-do-Chão, descrito inicialmente por Tancredi (1996) e por pesquisadores do Serviço Geológico do Brasil (VASQUEZ; SOUSA; CARVALHO 2008, MANOEL JR; MELO JR 2013). Os dados sobre o aquífero Alter do Chão ainda são incipientes e estudos tomaram maiores proporções a partir dos últimos dez anos segundo a Agência Nacional das Águas. O sistema aquífero Alter do Chão, apresenta dois sistemas principais: (i) parte superior, constituída por aquíferos livres, com menor profundidade, com espessura média de $50 \mathrm{~m}$ e localizado mais próximo ao lençol freático e a (ii) parte mais profunda, com espessura média de $430 \mathrm{~m}$ e denominado de aquífero confinado (TANCREDI 1996).

O consumo humano por águas subterrâneas é uma demanda crescente nas cidades da Amazônia, por ser opção economicamente viável frente ao processo acelerado de urbanização, por ser um recurso de boa qualidade natural que exige pouco tratamento sanitário e com baixo custo de captação.
Entretanto, a Organização Mundial da Saúde (OMS) vem alertando que mesmo a água sendo de origem subterrânea, poderá estar relacionada com a veiculação de agentes microbiológicos e compostos químicos que causam distúrbios e são determinantes em perfis epidemiológicos de saúde da população (Organização Mundial da Saúde 2017). Os fatores envolvidos na contaminação de águas subterrâneas podem ser de origem natural, devido a interação da água com rochas, ou de origem antrópica, em decorrência, por exemplo, de contaminação por dejetos sanitários e efluentes industriais que percolam em camadas do subsolo e atingem seções hídricas subterrâneas (ORGANIZAÇÃO MUNDIAL DA SAÚDE 2017).

Em avaliação pelo Instituto Trata Brasil no ano de 2020, de 100 municípios no Brasil a cidade de Santarém ocupou a $97^{\circ}$ posição em relação ao acesso a sistemas considerados satisfatórios de saneamento básico, incluindo a distribuição de água potável (Trata Brasil 2020). Segundo dados obtidos por Meschede (2018), estima-se que $61 \%$ da população de Santarém é servida pelo sistema de distribuição de água, operado pela Companhia de Saneamento do Pará (COSANPA), cuja captação pode alcançar até $250 \mathrm{~m}$ de profundidade, os residentes em bairros não atendidos pelo sistema COSANPA se valem de poços artesianos menos profundos observandose ainda o consumo de água engarrafada (MESCHEDE 2018).

$\mathrm{O}$ presente estudo contém informações sobre alguns parâmetros de qualidade e sobre a composição química de água subterrânea, utilizada para consumo humano, em duas estações do ano (seca e chuvosa) na cidade de Santarém. Apresenta uma discussão sobre as implicações para saúde do consumo de água com padrões de potabilidade alterados em Santarém e os resultados representam uma contribuição ao debate sobre as aglomerações urbanas e qualidade da água na Amazônia o qual vem ganhando importância crescente nos últimos anos. 


\section{MATERIAIS E MÉTODOS}

\section{1 ÁREA E LOCAL DE ESTUDO}

A área de estudo está situada no Oeste do estado do Pará, cidade de Santarém, na Amazônia central brasileira. A cidade de Santarém, com 304.589 mil habitantes estimados em 2019 (IBGE 2010), é a segunda região urbana mais importante do estado do Pará e está localizada no encontro dos rios Tapajós e Amazonas. A água para consumo em Santarém é de captação subterrânea, fornecida pela COSANPA, pela Prefeitura do município de Santarém ou através de perfurações particulares realizadas pelos próprios moradores (COSANPA 2013).

A região é marcada por dois períodos $(i)$ o período de seca (Julho a Novembro) e (ii) período chuvoso (Dezembro a Maio). O mês de junho pode ser considerado como de transição (Gomes et al. 2016), e, para esse estudo foi considerado dentro do período de seca. De acordo com os dados fornecidos pelo Instituto Nacional de Meteorologia (INMET), nos dois últimos anos, na região de Santarém, 95\% dos valores registrados para temperatura estiveram entre 22,5 a $32,7^{\circ} \mathrm{C}$ durante a estação seca e entre 22,3 a $31,2{ }^{\circ} \mathrm{C}$ durante a estação chuvosa (INMET 2018). Em 2019, a média mensal de precipitação e umidade relativa foram de 53,8 $\mathrm{mm}$ e $86,5 \%$ na estação seca e de $250,7 \mathrm{~mm}$ e de $91,2 \%$ na estação chuvosa, respectivamente (INMET 2020).

s locais de estudo foram definidos segundo os distritos urbanos de Santarém estabelecidos pelo IBGE, foram denominados nesse estudo de: Distrito 1 Nova República; Distrito 2 Alter do Chão; Distrito 3 Aldeia; Distrito 4 Prainha; Distrito 5 Maicá e Distrito 6 Santarenzinho. Em cada distrito selecionou-se 5 poços domiciliares de maneira aleatória e que apresentassem fonte de captação de água subterrânea. Na figura 1 apresenta-se os pontos de coleta de amostra no município de Santarém nos distritos.

\subsection{COLETA E ANÁLISE DAS AMOSTRAS DE ÁGUA}

Foram coletadas no total 60 amostras de água subterrânea, destinada ao consumo humano, em 30 domicílios distribuídos pelos distritos na cidade de Santarém durante o ano de 2018 e 2019 . Do total de amostras coletadas, 30 ocorreram durante um mês no período seco (novembro de 2018) e 30 durante um mês no período chuvoso (março de 2019). Utilizouse como ponto de coleta a torneira da cozinha de cada domicilio e coletou-se $100 \mathrm{ml}$ de amostra de água em um fraco estéril para a análise microbiológica, $100 \mathrm{ml}$ em frasco limpo para análise físico química, $50 \mathrm{ml} \mathrm{em} \mathrm{frasco}$ limpo para análise de nitrato e $50 \mathrm{ml}$ em frasco lavado previamente com ácido nítrico à $1 \%$ para análise de alumínio, todos providos de tampa. Após a coleta, as amostras foram mantidas refrigeradas em isopor com gelo com temperatura entre 4 a $8^{\circ} \mathrm{C}$ até o momento das análises.

As análises microbiológicas foram realizadas utilizando-se o método do Substrato Cromogênio Enzimático Colilert para a verificação de presença ou ausência de Coliformes Totais e Escherichia coli (E.coli) nas amostras de água. As amostras foram incubadas a $37^{\circ} \mathrm{C} \pm$ $3{ }^{\circ} \mathrm{C}$ por um período de 24 horas, seguindo as recomendações do Standard Methods for the
Examination of Water and Wastewater (APHA) de 2012. Considerou-se resultado positivo para coliformes totais amostras que apresentaram mudança em sua coloração para amarelo e para E.coli as amostras que foram submetidas a luz ultravioleta (UV) e que apresentaram fluorescência (Brasil 2011).

Para a determinação do Potencial Hidrogeniônico $(\mathrm{pH})$ foi utilizado um $\mathrm{pHmetro}$ portátil digital, modelo HI 991003 da marca Hanna ${ }^{\circledR}$ devidamente calibrado com soluções tamponadas ( $\mathrm{pH}$ de 4,0 e 7,0). Para a análise da turbidez expressa em Unidades Nefelométricas de Turbidez (NTU), utilizou-se um turbidímetro portátil modelo HI 98703 da marca Hanna ${ }^{\circledR}$, calibrado com soluções de 0 a 100 NTU.

As concentrações de nitrato $(\mathrm{mg} / \mathrm{L})$ e de alumínio $(\mathrm{mg} / \mathrm{L})$ nas amostras de água foram realizadas por um fotômetro multiparamétrico modelo HI 83099/COD da marca Hanna ${ }^{\circledR}$, que contém lâmpada de tungstênio com filtro de interferência de faixa estreita a $525 \mathrm{~nm}$. O equipamento determina concentrações entre 0 a $1,0 \mathrm{mg} / \mathrm{L}$ e entre 0 a $30 \mathrm{mg} / \mathrm{L}$ de alumínio e nitrato, respectivamente. Os equipamentos foram escolhidos por apresentarem limite de detecção compatíveis com os valores recomendados pelo Ministério da Saúde sobre pa- 


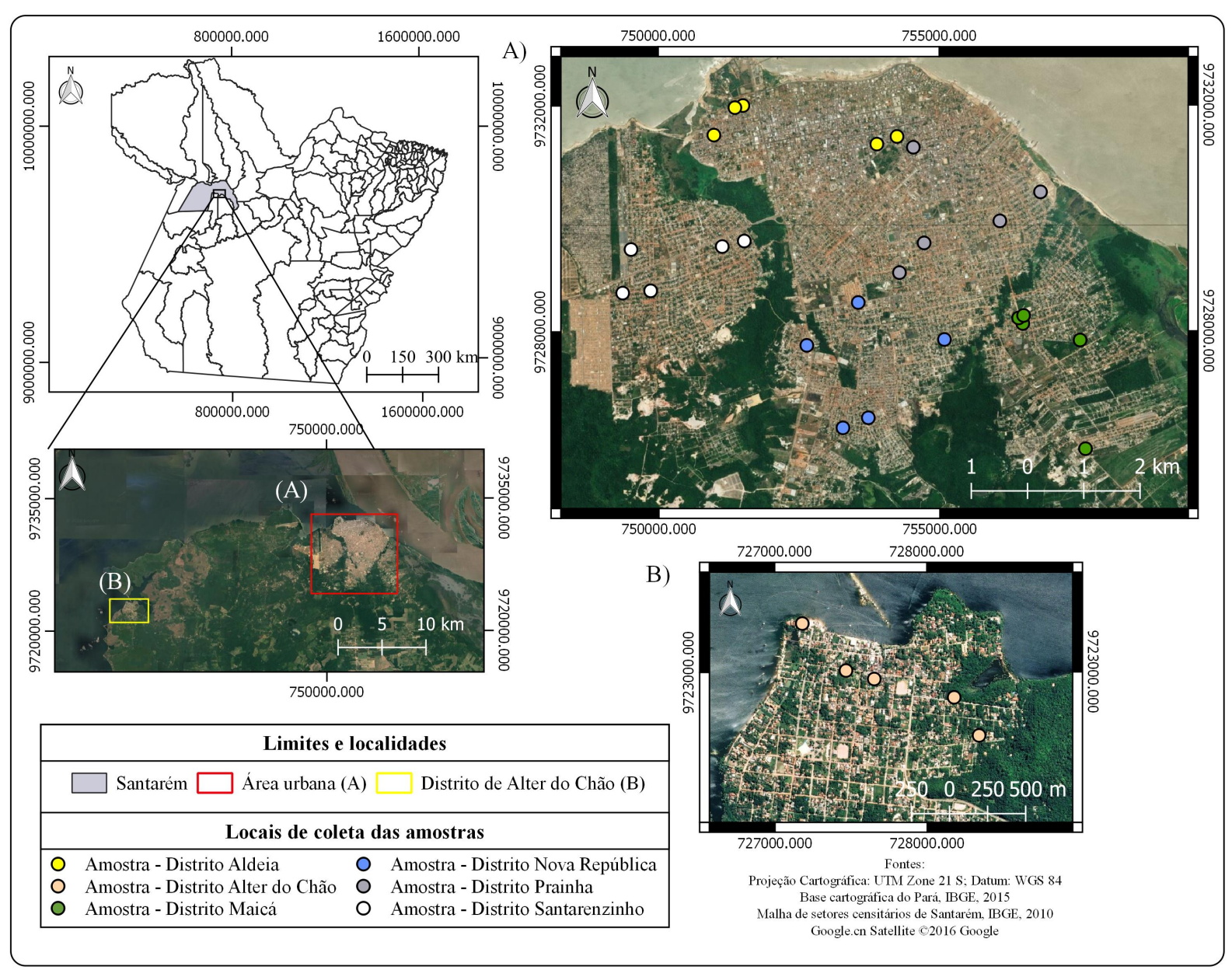

Figura 1 Localização dos poços em domicílios por distritos de coleta de água em Santarém, Oeste do Pará, Amazônia, Brasil.

drões de potabilidade de água para consumo humano (BRASIL 2016). Para as análises utilizou-se padrões de referência certificados.

As concentrações de mercúrio foram determinadas por espectrofotometria de absorção atômica, modelo DMA-80 Direct Mercury Analyzer, marca Milestone, automático sob fluxo de oxigênio, com limite de detecção de 5 ng/L de mercúrio. Para as análises utilizou-se padrões certificados de referência em hepatopâncreas de lagosta (TORT-2).

\subsection{ASPECTOS ÉTICOS}

O presente estudo obteve a aprovação do Comitê de Ética em Pesquisa da Universidade

\section{RESULTADOS E DISCUSSÃO}

\subsection{ANÁLISES MICROBIOLÓGICAS}

Os resultados apontaram contaminação por bactérias do grupo coliformes em $63 \%(n=19)$ e $70 \%(n=21)$ das amostras de água analisadas durante a estação seca e chuvosa, respectiva-
Os resultados foram tabulados no software Microsoft Office Excel (2013) e os parâmetros microbiológicos, físico-químicos e das concentrações químicas de nitrato, alumínio e mercúrio, foram comparados com as recomendações da Portaria do Ministério da Saúde de n²914 de 2011 (BRASIL 2011). A normalidade dos dados foi verificada por meio do teste de Shapiro-Wilk, após realizou-se o teste de Wilcoxon (Signed-rank test) bilateral para a comparação das medianas entre as estações seca e chuvosa no software Bioest5.3 (2012).

do Estado do Pará (UEPA) sob o parecer de 3.022.709. 


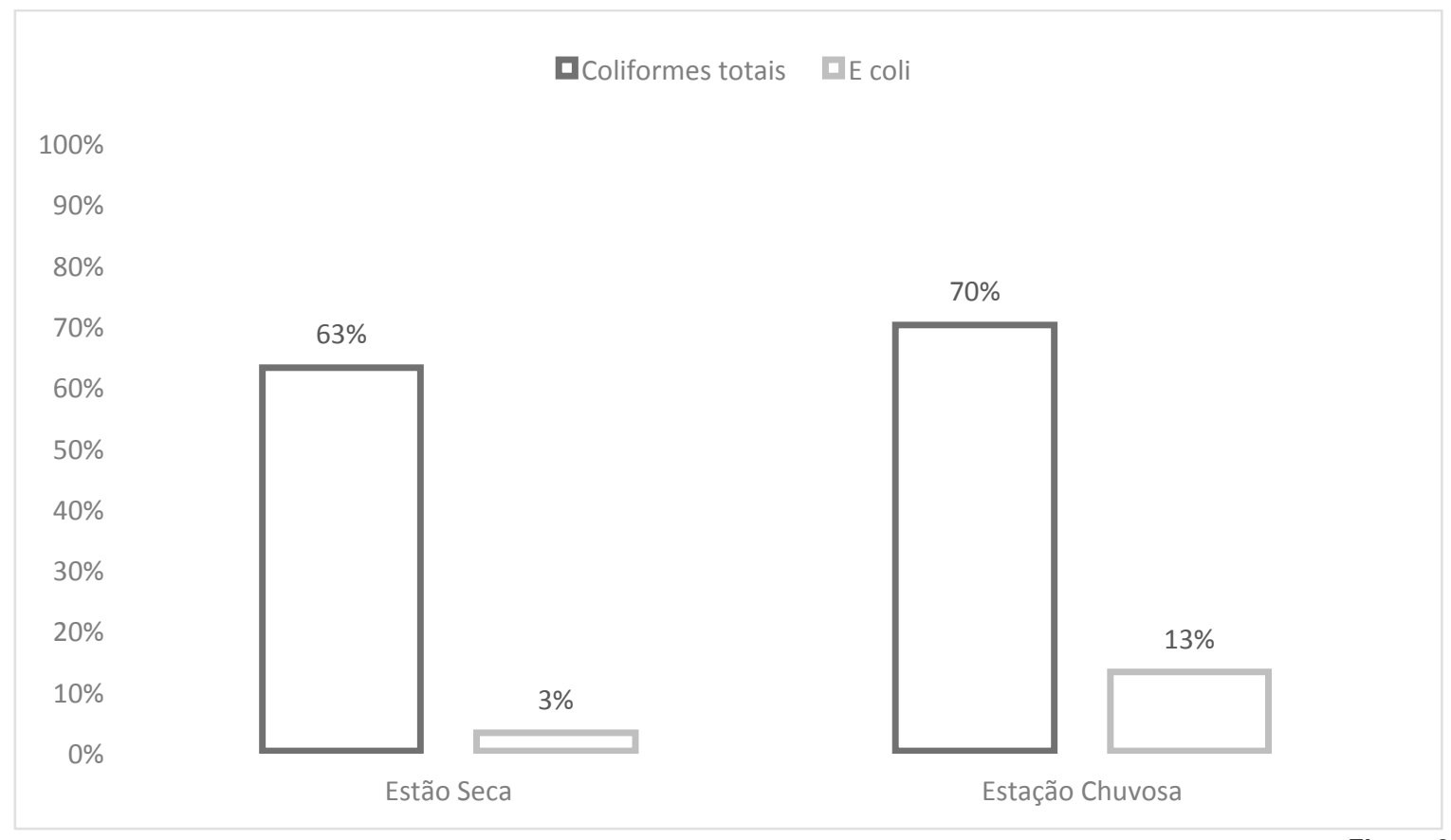

Percentual de amostras com crescimento para coliformes totais e E. coli em amostras de água coletadas em domicílios na região de Santarém, Oeste do Pará, Amazônia, Brasil

Em todos os distritos observou-se crescimento de coliformes totais, evidenciando não conformidade com a legislação, que determina a ausência desses microrganismos na água de consumo. Os dados são congruentes com os resultados obtidos por Meschede (2018), que também encontrou contaminação por coliformes totais em $78 \%$ de 36 análises de amostras de água realizadas em escolas públicas em Santarém, dentre as quais, $57 \%$ confirmaram presença de E. coli. Em outra pesquisa realizada por Mendes et al. (2017) em todas as amostras de água coletadas em 6 poços subterrâneos ao entorno de uma área desativada de despejo de resíduos sólidos em Santarém estiveram contaminadas por coliformes totais, entretanto, não houve confirmação para $E$. coli.

A presença desses microrganismos nas amostras do presente estudo, poderá ser atribuída a falta de manutenção adequada dos reservatórios domiciliares, devido à baixa profundidade dos poços (média de $34 \mathrm{~m}$ ), distância reduzida entre poço e fossas sépticas (média de $21 \mathrm{~m}$ ) e pela ausência de cloração e tratamento da água. A presença de coliformes totais e E.coli na água de consumo, devem ser vistas com cautela, uma vez que, poderão ser causadores de gastroenterites e outros distúrbios relacionados ao sistema gastrointestinal (Julião 2011). A presença de E. coli é um indicador de contaminação fecal e poderá indicar a coexistência de outros patógenos no ciclo oral fecal de transmissão de doenças de veiculação hídri- ca como febre tifoide, cólera, hepatite e parasitoses intestinais (OMS 2006).

Na Tabela 1 são apresentados os valores de $\mathrm{pH}$, turbidez, nitrato e alumínio obtidos durante as estações seca e chuvosa. Os testes de significância mostraram que não houve diferenças entre as concentrações obtidas no período seco e chuvoso $(p<0,05)$.

As concentrações de mercúrio encontraramse abaixo do limite de detecção (LD) para todas amostras analisadas, e por esse motivo não foram apresentadas na tabela. Embora pesquisas desenvolvidas na região de Santarém apontam concentrações elevadas de mercúrio em peixes e na água de rios (Bourdineand et al. 2015, Meneses 2016) para o presente estudo as amostras de água subterrânea mantiveram-se preservadas.

As águas mostraram-se ácidas nos domicílios avaliados com valores de $\mathrm{pH}$ variando de 3,53 a 5,94 durante as estações, em discordância com a legislação brasileira (Brasil 2011) que recomenda o consumo de água com $\mathrm{pH}$ entre 6,0 a 9,5 . Na região amazônica, estudos têm indicado baixo $\mathrm{pH}$ (cerca de 4,5 - 5,0) em águas, na sua maioria as de origem superficiais (Siqueira, Aprile; Migueis 2012, Aguiar, Peleja; Sousa 2014, Alves et al. 2012). Em águas subterrâneas, um número menor de estudos realizados na região Amazônica, apontaram também águas com baixo $\mathrm{pH}$. Medeiros et al. (2016) encontraram pH ácido em amostras de água $(4,0$ a 4,6) em ambas as estações, em 
Tabela 1 - Valores obtidos dos parâmetros físico-químicos e de composição química durante as estações seca e chuvosa para nitrato (mg/L); alumínio (mg/L); pH e turbidez (NTU) segundo o poço por distrito coletado em Santarém, Oeste do Pará, Amazônia, Brasil.

\begin{tabular}{|c|c|c|c|c|c|c|c|c|c|c|}
\hline \multirow{3}{*}{ Identificação } & \multirow[b]{3}{*}{ Poço } & \multirow[b]{3}{*}{$\begin{array}{l}\text { Profundidade } \\
\text { do poço* }(\mathrm{m})\end{array}$} & \multirow{2}{*}{\multicolumn{4}{|c|}{ Estação Seca }} & \multirow{2}{*}{\multicolumn{4}{|c|}{ Estação Chuvosa }} \\
\hline & & & & & & & & & & \\
\hline & & & $\mathrm{pH}$ & $\begin{array}{c}\text { turbidez } \\
\text { (NTU) }\end{array}$ & $\begin{array}{l}\text { nitrato } \\
(\mathrm{mg} / \mathrm{L})\end{array}$ & $\begin{array}{c}\mathrm{Al} \\
(\mathrm{mg} / \mathrm{L})\end{array}$ & $\mathrm{pH}$ & $\begin{array}{c}\text { turbidez } \\
\text { (NTU) }\end{array}$ & $\begin{array}{l}\text { nitrato } \\
(\mathrm{mg} / \mathrm{L})\end{array}$ & $\begin{array}{c}\mathrm{Al} \\
(\mathrm{mg} / \mathrm{L})\end{array}$ \\
\hline \multirow{5}{*}{$\begin{array}{c}\text { DISTRITO } 1 \\
\text { Nova República }\end{array}$} & D1-A & 24 & 4,20 & 0,32 & 5,40 & 0,03 & 4,15 & 0,12 & 6,0 & 0,15 \\
\hline & D1-B & 24 & 4,06 & 0,20 & 6,50 & 0,04 & 4,21 & 0,11 & 0,2 & 0,16 \\
\hline & D1-C & 48 & 4,54 & 0,21 & 0,80 & 0,09 & 4,65 & 0,11 & 0,2 & 0,01 \\
\hline & D1-D & 18 & 4,48 & 0,12 & 0,70 & 0,06 & 4,37 & 0,07 & 0,2 & 0,13 \\
\hline & D1-E & 20 & 3,84 & 0,22 & 2,70 & 0,33 & 3,70 & 0,10 & 19,4 & 0,24 \\
\hline \multirow{5}{*}{$\begin{array}{l}\text { DISTRITO } 2 \\
\text { Alter do Chão }\end{array}$} & D2-A & 24 & 5,86 & 0,15 & 2,72 & 0,11 & 4,52 & 0,65 & 7,3 & 0,36 \\
\hline & D2-B & 30 & 4,47 & 0,60 & 2,91 & 0,16 & 4,92 & 2,60 & 0,7 & 0,09 \\
\hline & $\mathrm{D} 2-\mathrm{C}$ & 24 & 4,18 & 0,09 & 3,70 & 0,09 & 4,88 & 0,09 & 0,5 & 0,12 \\
\hline & D2-D & 25 & 4,24 & 0,05 & 0,60 & 0,12 & 4,47 & 0,05 & 1,7 & 0,18 \\
\hline & D2-E & 30 & 4,21 & 0,12 & 3,30 & 0,18 & 4,27 & 0,11 & 7,3 & 0,13 \\
\hline \multirow{5}{*}{$\begin{array}{c}\text { DISTRITO } 3 \\
\text { Aldeia }\end{array}$} & D3-A & 30 & 3,72 & 0,22 & 30,00 & 0,27 & 4,16 & 0,33 & 18,5 & 0,19 \\
\hline & D3-B & 30 & 3,53 & 0,07 & 30,00 & 0,46 & 3,65 & 0,58 & 40,7 & 0,87 \\
\hline & D3-C & 20 & 3,84 & 6,16 & 13,0 & 0,32 & 3,93 & 6,60 & 14,9 & 0,28 \\
\hline & D3-D & 70 & 3,95 & 0,10 & 12,6 & 0,27 & 4,04 & 0,24 & 10,1 & 0,20 \\
\hline & D3-E & 25 & 3,84 & 0,10 & 10,70 & 0,38 & 3,82 & 0,14 & 7,6 & 1,85 \\
\hline \multirow{5}{*}{$\begin{array}{c}\text { DISTRITO } 4 \\
\text { Prainha }\end{array}$} & D4-A & 10 & 3,79 & 0,27 & 1,8 & 0,40 & 3,68 & 0,08 & 12,9 & 0,20 \\
\hline & D4-B & 100 & 4,73 & 0,11 & 3,00 & 0,12 & 4,54 & 0,19 & 0,7 & 0,14 \\
\hline & D4-C & 72 & 3,81 & 0,14 & 16,30 & 0,25 & 3,72 & 0,14 & 13,7 & 0,17 \\
\hline & D4-D & 50 & 3,76 & 0,06 & 14,50 & 0,14 & 3,87 & 0,37 & 16,3 & 0,82 \\
\hline & D4-E & 18 & 3,80 & 0,11 & 13,20 & 0,14 & 3,85 & 0,18 & 10,2 & 1,35 \\
\hline \multirow{5}{*}{$\begin{array}{c}\text { DISTRITO } 5 \\
\text { Maicá }\end{array}$} & D5-A & 24 & 5,60 & 0,36 & 5,10 & 0,14 & 5,94 & 0,85 & 9,6 & 0,04 \\
\hline & D5-B & 24 & 5,82 & 0,74 & 3,90 & 0,10 & 5,92 & 0,74 & 2,7 & 0,13 \\
\hline & D5-C & 24 & 4,97 & 0,08 & 3,70 & 0,11 & 4,90 & 0,11 & 6,9 & 0,07 \\
\hline & D5-D & 18 & 4,00 & 0,51 & 8,30 & 0,06 & 4,09 & 0,17 & 7,5 & 1,95 \\
\hline & D5-E & 50 & 4,76 & 0,09 & 0,40 & 0,14 & 4,51 & 0,07 & 0,2 & 0,01 \\
\hline \multirow{5}{*}{$\begin{array}{c}\text { DISTRITO } 6 \\
\text { Santarenzinho }\end{array}$} & D6-A & 54 & 4,20 & 0,14 & 2,40 & 0,18 & 4,34 & 0,32 & 2,4 & 0,12 \\
\hline & D6-B & 30 & 3,73 & 0,11 & 22,5 & 0,26 & 3,97 & 0,17 & 181 & 2,50 \\
\hline & D6-C & 63 & 3,79 & 0,06 & 18,0 & 0,36 & 3,92 & 0,11 & 139 & 1,60 \\
\hline & D6-D & 20 & 4,36 & 0,08 & 3,70 & 0,14 & 4,40 & 0,23 & 217 & 0,09 \\
\hline & D6-E & 38 & 4,03 & 0,10 & 9,40 & 0,33 & 5,02 & 0,19 & 0,2 & 0,03 \\
\hline Média & & & 4,27 & 0,4 & 8,39 & 0,19 & 4,34 & 0,52 & 25,18 & 0,47 \\
\hline Mediana & & & 4,12 & 0,12 & 4,50 & 0,14 & 4,24 & 0,17 & 7,40 & 0,16 \\
\hline Valor máximo & & & 5,82 & 6,16 & 16,30 & 0,38 & 5,92 & 6,60 & 217 & 2,50 \\
\hline Valor mínimo & & & 3,53 & 0,05 & 0,4 & 0,03 & 3,68 & 0,05 & 0,2 & 0,01 \\
\hline$p-$ valor $^{* *}$ & & & 0,0507 & 0,0656 & 0,3991 & 0,3991 & 0,0507 & 0,0656 & 0,3991 & 0,3991 \\
\hline Portaria $2914^{* * * *}$ & & & 6,0 a 9,5 & 0 a 5,0 & 0 a 10,0 & 0 a 0,2 & 6,0 a 9,5 & 0 a 5,0 & 0 a 10,0 & 0 a 0,2 \\
\hline
\end{tabular}

*Valor aproximado e informado pelo responsável do domicilio; **Teste de Wilcoxon (Signed-rank-test) bilateral $(p<0,05)$; ***Intervalo recomendado pela Portaria 2914

água proveniente de poços, consumida pelas comunidades ribeirinhas nos municípios de Abaetetuba e Barcarena, no leste do estado do Pará. Em outro estudo na Amazônia, Silva (2011) identificou pH da água entre 4,3 a 5,7 ao investigar as características hidroquímicas das água subterrânea na cidade de Manaus. Na região de Santarém, três estudos reportaram águas subterrâneas ácidas: (i) Tancredi (1996) encontrou valores de $\mathrm{pH}$ baixos para as amostras de água subterrânea variando de 4,0 a 4,7 em Santarém e em poços subterrâneos em localidades próximas; (ii) Nascimento, Fraia e Fenzl (2016) em estudo conduzido na cidade de Santarém, avaliaram água de poços domésticos privados município, e encontraram valores de $\mathrm{pH}$ situados entre 3,9 a 4,8 para águas de consumo e (iii) Mendes et al. (2017), avaliaram a qualidade físico química da água subterrânea de seis poços (profundidade entre $34 \mathrm{a}$ $78 \mathrm{~m}$ ) no bairro Santo André em Santarém, e encontraram valores de $\mathrm{pH}$ entre 3,7 a 5,6.

A presença de materiais siliclásticos nas rochas da formação Alter do Chão contribuem para a determinação da acidez na água do aquífero de forma natural (MENDES et al. 2017).
Os efeitos diretos em saúde dos consumidores ainda permanecem incertos. Faltam estudos de cunho experimental disponíveis até o momento na literatura. A ingestão de bebidas ácidas em geral aumentam a possibilidade de alterações e diminuição do $\mathrm{pH}$ duodenal e a redução significativa dos períodos de alcalinização duodenal (MCCLOY; GRENBERG; BARON 1984). Além disso, um efeito indireto à saúde humana poderá ser associado ao $\mathrm{pH}$ baixo das águas, devido a uma solubilização aumentada de minerais em água ácida, com a liberação, por exemplo, de $\mathrm{Al}, \mathrm{Cd}$ e $\mathrm{Pb}$ o que pode resultar em concentrações mais altas desses elementos químicos em água de consumo (OMS 2003).

A turbidez apresentou valores abaixo do permitido pela legislação vigente (Brasil 2011), com a exceção, de um ponto que superou o limite em até 1,2 vezes na estação seca e 1,3 vezes durante o período chuvoso. O parâmetro turbidez não representa efeitos diretos em saúde, mas torna-se uma medida complementar de potabilidade da água por indicar a presença de materiais sólidos em suspensão na água de consumo (OMS 2006). 
Os resultados mostraram que $33 \%(\mathrm{n}=10)$ e $40 \%(n=12)$ das amostras analisadas no período seco e chuvoso estiveram com níveis de nitrato excedentes ao valor de $10 \mathrm{mg} / \mathrm{L}$ recomendado pela legislação brasileira (Brasil 2011), respectivamente. O nitrato que poderá ser encontrado em mananciais subterrâneos, trata-se de um poluente inorgânico com alta mobilidade, de fontes de atividades antrópicas, com origem em fertilizantes nitrogenados, adubos orgânicos, dejetos humanos, animais e efluentes industriais (MAJUMDAR; GPTA 2000). Altos níveis de nitrato sugerem condições sanitárias insatisfatórias, devido ser resultante dos estágios finais de decomposição da matéria orgânica e contaminação da água subterrânea pode ocorrer devido a sua infiltração nas camadas do subsolo, podendo ter correlação com a baixa profundidade dos poços e densidade populacional (VANIER et al. 2016).

Além de níveis excessivos de nitrato, observou-se que em $37 \%(n=11)$ e $33 \%(n=10)$ das amostras avaliadas mostraram concentrações superior ao recomendado de alumínio (acima de 0,2 mg/L) durante as estações seca e chuvosa, respectivamente. Os solos ricos em matéria orgânica e ácidos são fatores contribuintes à mobilidade e à solubilidade do alumínio nas águas subterrâneas (BONDY 2016). Os achados do presente estudo são coerentes com

\section{CONCLUSÃO}

Em conjunto, os resultados dessa pesquisa, indicam que a água consumida nos domicílios proveniente de poços em Santarém, apresentase com alguns parâmetros de qualidade em desacordo com os estabelecidos pela na Portaria 2914 do Ministério da Saúde.

Os poços domiciliares analisados, embora de captação subterrânea, estão sujeitos à incorporação de poluentes como o nitrato, alumínio e a contaminação por bactérias fecais devido a sua pouca profundidade e influência antrópica como, por exemplo, das fossas sépticas, acentuadamente durante a estação chuvosa. Os moradores de Santarém ao consumirem a água com presença de bactérias do grupo dos coliformes estão mais suscetíveis a adoecimentos intestinais. A ingestão através da água de nitrato pode ser associada a vários efeitos prejudiciais aos seres humanos, como o risco aumen-

\section{AGRADECIMENTOS}

A Universidade Federal do Oeste do Pará (UFOPA) por meio do Programa Institucional de Bolsas de Iniciação Científicas (PIBIC) e ao os dados reportados por Nascimento, Fraia e Fenzl (2016) que avaliaram as características químicas de águas subterrâneas provenientes de poços domésticos em Santarém, e encontraram níveis elevados (acima de $200 \mathrm{ng} / \mathrm{mL}$ ) de $\mathrm{Al}$ em 06 das 09 amostras de água analisadas. Em outro estudo, Meschede (2018), também, encontrou concentrações de Al superiores ao recomendado pela Portaria 2914 em 16 amostras de água das 36 coletadas em escolas localizadas no município de Santarém e Mojuí dos Campos.

Estudos têm demonstrado a influência, ainda em debate, sobre o efeito da exposição crônica ao alumínio através da água de consumo como fator desencadeante de encefalopatias, tais como demência e doença de Alzheimer (Bondy 2016, Walton 2014). Ressalta-se que existem estudos na literatura que não sustentam essa relação entre ingestão de altas concentrações de alumínio através da água e o desenvolvimento de doenças neurológicas sendo que algumas explicações são propostas: (i) o alumínio mesmo estando em altas concentrações na água, irá contribuir apenas com uma fração da dieta total ingerida pelo humano e (ii) o alumínio é muito pouco absorvido pela via gástrica intestinal, menos do que $1 \%$ (ATSDR 2008, OMS 2010).

tado a metahemoglobinemia infantil e o desenvolvimento de alterações do trato digestivo. A presença de elevadas concentrações de alumínio em algumas amostras deverá ser investigada por novos estudos, uma vez que, não se descarta a sua relação com efeitos neurológicos a longo prazo. Os resultados do presente estudo, evidenciaram que a água de consumo é ácida em todas as amostras coletadas. Deve ser levado em conta, que outros estudos na Amazônia pontuaram sobre o consumo de água ácida, o que parece não ser uma característica exclusiva de Santarém.

Os riscos à saúde associados ao consumo de água com a qualidade comprometida poderão ser minimizados através do seu monitoramento periódico nesses bairros e a adoção de práticas de saneamento e abastecimento hídrico compatíveis com o rápido crescimento da cidade. 


\section{REFERÊNCIAS}

ATSDR - Agency for Toxic Substances and Disease Registry. 2008. Toxicological profile for Aluminium. U.S. Department of Health and Human Services, Public Health Service Agency for Toxic Substances and Disease Registry: Atlanta.

AGUiAR, C. P. O.; PELEJA, J. R. P.; SOUSA K. N. S. 2014. Qualidade da Água em Microbacias hidrográficas com agricultura Nos municípios de Santarém e Belterra, Pará. Revista Árvore, 38, 983-992.

ALVES, L. C. C.; EL-ROBRINI, M.; SANTOS, M. L. S.; MONTEIRO, S. M.; BARBOSA, L. P. F.; GUIMARÃES, J. T. 2012. Qualidade das águas superficiais e avaliação do estado trófico do rio Arari (Ilha de Marajó, norte do Brasil). Acta Amazônica, 42, 115-124.

APHA - American Public Health Association. 2012. Standard Methods for the Examination of Water and Wastewater. 22nd Ed. Washington DC.

BONDY, S. C. 2016. Low levels of aluminum can lead to behavioral and morphological changes associated with Alzheime's disease and agerelated neurodegeneration. Neurotoxicology, 222-229.

BOURDINEAUD, J. P.; DURRIEU, G.; SARRAZIN, S. L.; SILVA, W. C.; MOURÃO, R. H.; OLIVEIRA, R. B. 2015. Mercurial exposure of residents of Santarém and Oriximiná cities (Pará, Brazil) through fish consumption. Environ Sci Pollut Res Int., 22:12150-61.

BRASIL. 2016. Manual prático de análise de água. Brasília: Fundação Nacional de Saúde (Funasa), 2: $146 \mathrm{p}$

BRASIL. 2011. Portaria n. ${ }^{\circ} 2.914$, de 12 de Dezembro de 2011. Dispõe sobre normas de potabilidade de água para o consumo humano. Ministério da Saúde - Oficial da União.

COSANPA - Companhia de Saneamento do Pará. 2013. Histórico Disponível em: http://www.cosanpa.pa.gov.br. Acessado em 20 de mar 2020.

CORTÊS, J. C. 2012. Mobilidade e distribuição populacional em Santarém, Pará: recente reconfiguração do meio rural na Amazônia. Dissertação de Mestrado em Filosofia, Universidade Estadual de Campinas, $162 \mathrm{p}$.

GOMES, A. C. S.; COSTA, G. B.; VALE, R. S., SANTANA R. A. S., BATALHA S. S. A., SILVA J. T., FITZJARRALD D. R. 2016. Índices micrometeorológicos e internações de crianças por doenças respiratórias em Santarém, Oeste do Pará. Ciência e Natura, 38:1-6.

IBGE - Instituto Nacional de Geografia e Estatística. 2010. Cidades. Censo 2010. Disponível em: https://cidades.ibge.gov.br/brasil/pa/santarem/pa norama. Acessado em: 12 abr 2020.

INMET - Instituto Nacional de Meteorologia. 2020. Série Histórica - mensal. Estação meteorológica de Belterra - PA. Disponível em http://www.inmet.gov.br/projetos/rede/pesquisa/ ge-

ra_serie_txt_mensal.php?\&mRelEstacao $=82246$ $\& \bar{b}$ tnProcesso $=$ serie $\&$ mRelDtInicio $=01 / 01 / 2018$ $\&$ mRelDtFim $=31 / 12 / 2019 \& \mathrm{mAtributos}=,,,,,,,, 1$, $1,,, 1,, 1,1$, . Acessado em 12 de jun 2020.

INSTITUTO TRATA BRASIL. 2020. Organização da Sociedade Civil de Interesse Público. Ranking do Saneamento. Disponível em: http://ratabrasil.com.br/images/estudos/itb/rank ing_2020/Tabela_100_cidades_Ranking_Sanea mento_4.pdf. Acessado em 09 abr 2020.

JULIÃO, F. C. Avaliação das condições microbiológicas e físico químicas da água de reservatório domiciliar e predial: importância da qualidade dessa água no contexto da saúde pública. Tese de Doutorado em Saúde Pública. Escola de Enfermagem de Ribeirão Preto. 2011.

MAJUMDAR, D.; GUPTA, N. Nitrate pollution of groundwater and associated human health disorders. 2000. Indian Journal of Environmental Health, 42:28-39.

MANOEL JR., I.; MELO JR., H. R. 2013. Aquífero Alter do Chão: Análises Químicas In Loco dos Poços Construídos pela RIMAS (SGB-CPRM). In: XX Simpósio Brasileiro de Recursos Hídricos.

MCCLOY, R. F.; GRENBERG, G. R.; BARON, J. H. 1984. Duodenal $\mathrm{pH}$ in health and duodenal ulcer disease: effect og a meal, Coca-Cola, smoking and cimetidine. Gut., 25: 386-392.

MEDEIROS, A. C.; LIMA, M.; GUIMARAES, R. M. 2016. Avaliação da qualidade da água de consumo por comunidades ribeirinhas em áreas de exposição a poluentes urbanos e industriais nos municípios de Abaetetuba e Barcarena no estado do Pará, Brasil. Ciência Saúde Coletiva, 21: 695-708.

MENDES, A.; GALVÃO, P.; SOUSA, J.; SILVA, I.; CARNEIRO, R. N. 2017. Relations of the groundwater quality and disorderly occupation in an Amazon low income neighbourhood developed over a former dump area, Santarém-PA, Brazil. Environment, Development and Sustainability, 21:1-16.

MENESES, H. N. M. 2016. Efeito de genes da família glutationa s-transferase em uma população do baixo amazonas ambientalmente expostas ao mercúrio. Tese de Doutorado em Sociedade, Natureza e Desenvolvimento. Universidade Federal do Oeste do Pará, 112 p.

MESCHEDE, M. S. C. 2018. Implicações para a saúde de escolares a partir do consumo de água e material particulado atmosférico inalado em escolas de Santarém e Mojuí dos Campos, Pará, Amazônia. Tese de Doutorado em Sociedade, Natureza e Desenvolvimento. Universidade Federal do Oeste do Pará, 165 p.

NASCIMENTO, F. S.; FRAIA, R. S.; FENZL, N. 2016. Caracterização química da água potável do município de Santarém, estado do Pará: uma 
análise comparativa. In: XIII Congresso de Geoquímica dos Países de Língua Portuguesa, Fortaleza.

OLIVEIRA, J. M. G. 2008. Expansão urbana e periferização de Santarém-PA, Brasil: Questões para o planejamento urbano. In: X Colóquio Internacional de Geocrítica. Universidade de Barcelona. Barcelona.

OMS - Organização Mundial da Saúde. 2003. pH in Drinking - Water. WHO Library Cataloguing in Publication Data, Geneva.

OMS - Organização Mundial da Saúde. Protecting Groundwater for Health - Managing the quality of drinking water sources. IWA Publishing, London, 2006.

OMS - Organização Mundial da Saúde. 2010. Aluminum in drinking water - background document for development of WHO guidelines for drinking water quality. WHO Library Cataloguing in Publication Data, Geneva.

OMS - Organização Mundial da Saúde. 2017. Guidelines for drinking water quality: fourth edition incorporating the first addendum. WHO Library Cataloguing in Publication Data, Geneva.

SIQUEIRA, G. W; APRILE, F.; MIGUÉIS, A. M. 2012. Diagnóstico da qualidade da água do rio
Parauapebas (Pará - Brasil). Acta Amazônica, 42: $413-422$.

SILVA, M. L. 2011. Características das águas subterrâneas numa faixa norte-sul na cidade de Manaus (AM). Revista Escola Minas (online,) 54: 115-120.

TANCREDI, A. C. F. N. S. 1996. Recursos hídricos subterrâneos de Santarém: fundamentos para uso e proteção. Tese de Doutorado em Geologia. Universidade Federal do Pará (UFPA), $146 \mathrm{p}$.

VANIER, C.; FIGUEIREDO, G. M.; ODA, G. H.; IRITANI, M. A.; FERREIRA, L.; TAVARES, T.; ALBUQUERQUE, J. L. 2016. Ocorrência de Nitrato no sistema aquífero Bauru e sua relação com a ocupação urbana no município de Marilia (SP). In: XIX Congresso Brasileiro de Águas Subterrâneas. Campinas-SP.

VASQUEZ, M. L.; SOUSA, C. S.; CARVALHO, J. M. A. 2008. Mapa Geológico e de Recursos Minerais do Estado do Pará, escala 1:1.000.000, CPRM - Serviço Geológico do Brasil, Superintendência Regional de Belém.

WALTON, J. R. 2014. Chronic Aluminium Intake Causes Alzheimer's Disease: Applying Sir Austin Bradford Hill's Causality Criteria. Journal of Alzheimer's Disease, 40:765-838. 